\title{
Using Time-Frequency Analysis Technique in the Classification of Surface EMG Signals
}

\author{
Gwo-Ching Jang, Cheng-Kung Cheng*, Jin-Shin Lai**, and Te-Son Kuo \\ Department of Electrical Engineering, ${ }^{*}$ Center for Biomedical \\ Engineering. and ${ }^{* *}$ Department of Rehabilitation, National Taiwan \\ University, R.O.C.
}

\begin{abstract}
A time-frequency analysis technique with Wigner-Ville distribution was developed for discriminating a set of upper limb motions using just one channel surface EMG signals. EMG data were obtained from the distal portion of the deltoid. The limb motions included elbow extension/flexion, wrist pronation/supination, and wrist abduction/adductiion. The $2 \mathrm{D}$ cross-correlation was employed for classification of the upper limb functions.
\end{abstract}

\section{Introduction}

Electromyographic (E.MG) signals from body's intac$t$ musculacure have been suggested and utilized by many researchers as various trigger conmands for a multifunction lower arm prosthesis[1][2]. The goal of this research is to deal with the pattern recognition of EMG signals using time-frequency analysis technique to generate control commands for prosthetic devices. A single channel EMG signals were measured from the distal portion of the deltoid to discriminate six limb motions included elbow extension/flexion, wrist pronation/supination, and wrist abduction/adduction[3].

The spectral characteristics of the E.IG signals concerning with different contractions of the same muscle is different [2]. This phenomenon implied that discriminatory information was contained in the difference between the spectral characteristics varieties for different muscle activation patterns at the same muscle site. The timefrequency analysis is to describe situations where the frequency content of a signal is changing in time. The classification technique based on time-frequency analysis has the greater potential for formation of the feature vector which is afforded by the 2D nature of representations[4]. In this paper, EMG pattern recognition using time-frequency analysis based on Wigner-Ville distribution (WVD) was considered. The 2D cross-correlation was employed for classification of the upper limb motions.

\section{Methods}

\section{Wigner-Ville Distribution}

The time-frequency representation of WVD provided high resolution signal characterization in a time-frequency space, and good noise rejection performance [4]. The realvalued, discrete WVD representation is defined as follow

$$
W_{x}(k, l)=2 \sum_{m=-K}^{K} x(k+m) x^{*}(k-m) e^{-j \frac{2 \pi 1}{\eta} l m}
$$

where $k, l$ are the time-index and the frequency-index, respectively, and the length of interest signals is $N=2 K+1$.

\section{EMG Processing}

Twenty sets of EMG data were recorded from distal portion of deltoid. A preamplifier with a bandpass filter between $10 \mathrm{~Hz}$ and $1 \mathrm{kHz}$ was used to reduce the effects of high frequency noise and motion artifact. The analog-todigital converter, having 12-bit resolution, was used to sample the amplified EMG signals at $2 \mathrm{kHz}$. The timefrequency analysis was applied with WVD to generate $2 \mathrm{D}$ feature vectors. In order to reduce the wide dynamic range of WVD of EMG signals, the logarithmic compression scheme was employed. The feature vector was normalized with respect to its root-mean-square valus. The $2 \mathrm{D}$ cross-correlation method was used to classify the upper limb motions. All the computing operations were computered by the Texas Instruments T.MS320C30 floating-point digital signal processor. 


\section{Results and Discussions}

The WVD of EMG signals for wrist abduction and adduction movement is shown in Fig. 1. Note that the timefrequency distribution is defferent as wrist abduction and adduction in the Fig. 1. Based on this difference between the spectral characteristics varieties, the limb motions can be classify. The preliminary discrimination rate results derived from $2 \mathrm{D}$ cross-correlation technique is shown in Table I. These recognition rates show that the WVD performs well in generating control commands for prosthetic devices. This time-frequency analysis technique can also be used in studying muscle fatigue in the futu:e.

\section{$\underline{\text { References }}$}

[1]. Graupe D., Salahi J., Zhang D.,"Stochastic Analysis of Myoelectric Temporal Signatures for Multifunctional Single-Site Activation of Prostheses and Orthoses,"J Biomed. Eng., $7: 18-29,1985$.

[2]. Doerschuk PC., Gustafson DE., Willsky AS., "Upper Extremity Limb Function Discrimination Using EMG Signal Analysis," IEEE Trans. Biomed. Eng. $30: 18-28,1983$.

[3]. Michael F. Kelly, Philip A. Parker, and Robert N. Scott, "Myoelectric Signal Analysis Using Neural Networks," IEEE Eng. Medicine \& Biology Mag., pp. 61-64, March 1990.

[4]. Boualem B. and Peter O.:"A Methodology for Detection and Classification of Some Linderwater Acoustic Signals Uising Time-Frequency Analysis Techniques,"IEEE Trans. Acoust. Speech Signal Processing, vol. 38. no. 11, pp. 1829-1841, Nov. 1990.

Table I. The recognition rate for six limb motions

\begin{tabular}{|cc|c|}
\hline Wrist & Supination & $95 \%$ \\
\hline Wrist & Pronation & $90 \%$ \\
\hline Elbow & Extenstion & $70 \%$ \\
\hline Elbow & Flexion & $90 \%$ \\
\hline Wrist & Abduction & $100 \%$ \\
\hline Wrist & Adduction & $80 \%$ \\
\hline
\end{tabular}

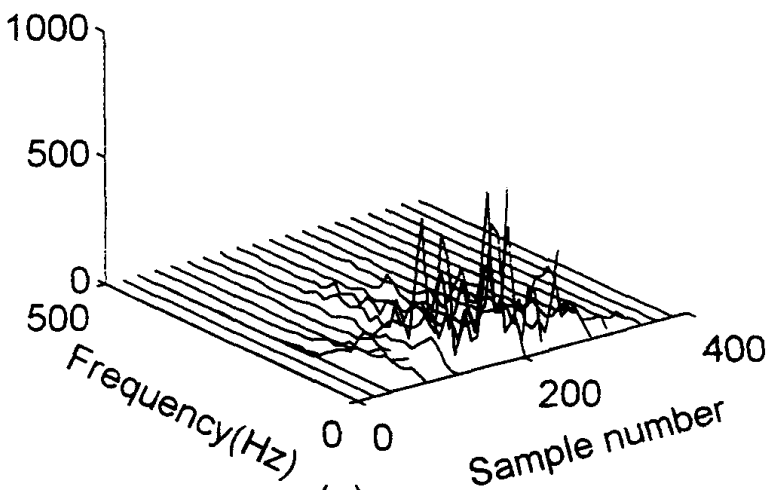

(a)

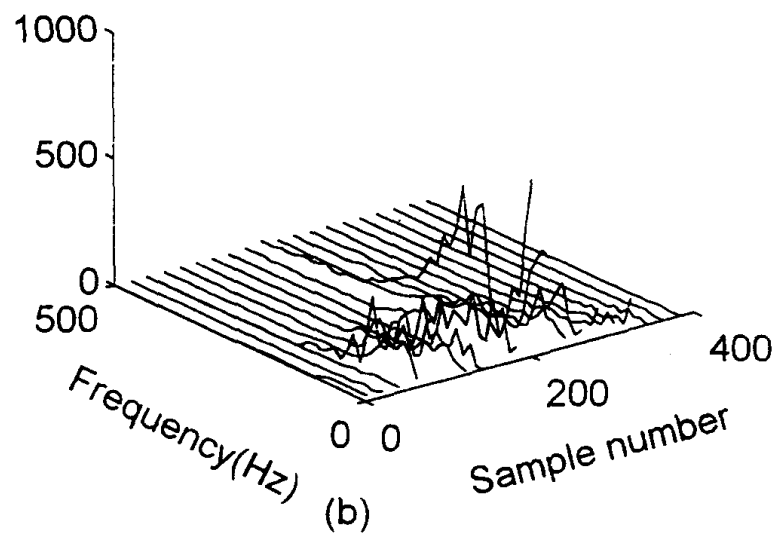

Figure 1. The WVD of EMG signals for (a) wrist abduction (b) wrist adduction 